\title{
Peroxiredoxin of Arthrospira platensis derived short molecule YT12 influences antioxidant and anticancer activity
}

\begin{abstract}
This study demonstrates both the antioxidant and anticancer potential of the novel short molecule YT12 derived from peroxiredoxin (Prx) of spirulina, Arthrospira platensis (Ap). ApPrx showed significant reduction in reactive oxygen species (ROS) against hydrogen peroxide (H2O2) stress. The complementary DNA sequence of ApPrx contained 706 nucleotides and its coding region possessed 546 nucleotides between position 115 and 660 . Real-time quantitative reverse transcription polymerase chain reaction analysis confirmed the messenger RNA expression of ApPrx due to $\mathrm{H} 2 \mathrm{O} 2$ exposure in spirulina cells at regular intervals, in which the highest expression was noticed on Day 20. Cytotoxicity assay was performed using human peripheral blood mononuclear cells, and revealed that at $10 \mu \mathrm{M}$, the YT12 did not exhibit any notable toxicity. Furthermore, ROS scavenging activity of YT12 was performed using DCF-DA assay, in which YT12 scavenged a significant amount of ROS at $25 \mu \mathrm{M}$ in H2O2-treated blood leukocytes. The intracellular ROS in human colon adenocarcinoma cells (HT-29) was regulated by oxidative stress, where the YT12 scavenges ROS in HT-29 cells at $12.5 \mu \mathrm{M}$. Findings show that YT12 peptide has anticancer activity, when treated against HT-29 cells. Through the MTT assay, YT12 showed vital cytotoxicity against HT-29 cells. These finding suggested that YT12 is a potent antioxidant molecule which defends ROS against oxidative stress and plays a role in redox balance.
\end{abstract}

Keyword: Arthrospira platensis; Antioxidant peptide; Peroxiredoxin; Cytotoxicity; Anticancer 